\title{
Determinação da viscosidade por meio da velocidade terminal: uso da força de arrasto com termo quadrático na velocidade
}

Determination of viscosity through terminal velocity: use of the drag force with a quadratic term in velocity

\author{
Lev Vertchenko*1, Larissa Vertchenko²
}

\author{
${ }^{1}$ Departamento de Física e Química, Pontifícia, Universidade Católica de Minas Gerais, Belo Horizonte, MG, Brasil \\ ${ }^{2}$ Department of Photonics Engineering, Technical University of, Denmark, Ørsteds Plads 343 DK-2800 Kgs. Lyngby, Denmark
}

Recebido em 05 de Fevereiro, 2017. Revisado em 19 de Março, 2017. Aceito em 04 de Abril, 2017.

\begin{abstract}
Propomos a correção, por um fator adimensional, do termo quadrático na velocidade da força de arrasto de Oseen para que ela seja usada na determinação do coeficiente de viscosidade da glicerina através da medição da velocidade terminal de esferas em queda dentro do fluido. Esse fator incorpora o efeito da parede do recipiente sobre o movimento das esferas, analogamente à correção da força de Stokes pelo fator de Ladenburg, e permite compatibilizar a força de Oseen com os dados experimentais do coeficiente de arrasto até números de Reynolds próximos a 30. Admitindo uma relação de proporcionalidade entre esses fatores, o coeficiente de viscosidade passa a ser o coeficiente linear na relação entre a viscosidade que seria obtida se fosse considerada apenas a força de Stokes e o produto da velocidade terminal pelo diâmetro das esferas. A experiência com esferas de aço em queda dentro de tubos de diferentes diâmetros forneceu valores para o coeficiente de viscosidade compatíveis com a temperatura em que se trabalhou. Esse método pôde ser aplicado até números de Reynolds da ordem da dezena, relaxando a restrição de se trabalhar com números de Reynolds inferiores a 0,5 na determinação da viscosidade através do método de Stokes.
\end{abstract}

Palavras-chave: Força de arrasto, viscosidade, número de Reynolds.

A correction to the term with quadratic dependency of the velocity in the Oseen's drag force by a dimensionless factor is proposed in order to determine the viscosity of glycerin through the measurement of the terminal velocity of spheres falling inside the fluid. This factor incorporates the effect of the container's wall over the movement of the spheres, analogously to the correction of the Stoke's force by the Landenburg factor, and permits to make the Oseen's force compatible with the experimental data for the drag coefficient up to Reynolds number near 30 . Admitting a proportionality relation between these factors, the viscosity coefficient turns into a linear coefficient in the relation between the parameter that corresponds to the viscosity that would be determined if only the Stokes' force was considered and the product of the terminal velocity by the diameter of the spheres. The experiment with steel spheres falling inside tubes of different diameters showed values for the viscosity coefficient of glycerin compatible with the expected, for the temperature range worked. This method might be applied to Reynolds number of order of ten of the sphere's motion, relaxing the restriction of working with Reynolds numbers inferior to 0.5 for the determination of the viscosity through the Stokes' method.

Keywords: Drag force, viscosity, Reynolds number.

\section{Introdução}

A viscosidade está associada a uma espécie de atrito interno que causa friç̧ão entre as camadas do fluido que se movimentam com velocidades diferentes e é explicada, ao nível microscópico, como devendo-se à transferência de momento linear entre as partículas que compõem o fluido. Tecnicamente ela é caracterizada pelo coeficiente de viscosidade dinâmico, $\eta$, definido como a razão entre a força de cisalhamento, por unidade de área, à qual o fluido é submetido, e o gradiente de velocidade perpendicular a essa força, que dela decorre. A determinação

*Endereço de correspondência: vertlar25@gmail.com do coeficiente de viscosidade de fluidos tem enorme importância nas mais diversas áreas, desde a engenharia, caracterizando fluidos lubrificantes de máquinas, dentre muitos exemplos, até a medicina, relacionando-se à secreção de muco do organismo, à circulação sanguínea, à distribuição de partículas de fármacos por meio desta, etc.

Uma prática de laboratório muito comum nas disciplinas de Física Geral de cursos universitários consiste na determinação da viscosidade da glicerina deixando cair esferas de ferro ou aço dentro de um recipiente cilíndrico contendo esse fluido e medindo as suas velocidades terminais, que ocorrem quando os seus pesos são equilibrados 
pelo empuxo e pela força de arrasto do fluido. Usualmente emprega-se para essa força de arrasto a expressão obtida por George Gabriel Stokes em 1851 [1], em que a força viscosa é proporcional à primeira potência da velocidade. Escolhe-se trabalhar com glicerina pelo fato dessa força viscosa, em temperaturas ambiente normais, ser o termo dominante na força de arrasto, cuja expressão é corrigida por termos que envolvem potências superiores da velocidade, e pela sua transparência, que facilita a determinação da velocidade das esferas através de sensores óticos. A opção pelo uso de esferas de ferro ou aço permite que as mesmas sejam resgatadas do fundo da coluna de fluido com o auxílio de um magneto.

Os estudantes se surpreendem que o cálculo do coeficiente de viscosidade fornece valores dependentes do tamanho das esferas usadas, o que não é razoável, uma vez que as esferas são empregadas apenas como sondas, enquanto a viscosidade é uma propriedade do fluido. Esse método para a obtenção do coeficiente de viscosidade foi analisado por Sandoval, Caram e Salinas [2], doravante SCS, que incorporaram a influência do diâmetro da coluna sobre o movimento das esferas e corroboraram a advertência de que a expressão da força de Stokes somente é adequada às esferas muito pequenas, de menor velocidade terminal, cujo movimento no fluido é tecnicamente caracterizado por números de Reynolds, definidos adiante, inferiores a 0,5 [3]. Soares et al. [4] também usaram a força de Stokes no estudo da queda de esferas em um meio viscoso para baixos números de Reynolds, mas propuseram que à massa da esfera fosse adicionada a massa do fluido por ela arrastada. Essa massa de fluido arrastada é difícil de ser quantificada em virtude da variação da velocidade do fluido em torno da esfera e, então, serviu de parâmetro de ajuste aos dados experimentais da equação de posição da esfera em função do tempo decorrente do modelo, permitindo a determinação da viscosidade.

Propomos aqui um procedimento para a determinação do coeficiente de viscosidade por meio da medição da velocidade terminal que relaxa essa restrição aos números de Reynolds, ao acrescentar um termo de dependência quadrática da velocidade na força de arrasto, admitindo uma correção na formulação de Carl Wilhelm Oseen, de 1910 [5]. Com isso não ocorre alteração na parte experimental, mas apenas o acréscimo de um simples procedimento no tratamento dos dados coletados. $\mathrm{Na}$ perspectiva do ensino de Física Geral, isso é positivo, pois permite uma discussão de forças de arrasto com dependência quadrática da velocidade, que são mencionadas nos tradicionais manuais de Física básica [6] e textos de Mecânica de nível intermediário [7, 8] que, porém, desenvolvem a análise do movimento do objeto separando os casos da força com dependência linear ou quadrática, enquanto apontamos para uma situação que necessita usar conjuntamente as duas formas de dependência. A tendência de tratar separadamente os casos reflete-se, inclusive, nos experimentos que são propostos para o la- boratório didático com o objetivo de distinguir as forças de arrasto pela dependência linear ou quadrática da velocidade. Takahashi e Thompson [9] propõem analisar o dimensionamento da massa efetiva (associada à combinação do empuxo com o peso) de um balão de ar em queda em relação à sua velocidade terminal para concluir pela atuação de uma força de arrasto quadrática na velocidade. De forma semelhante, Owen e Ryu [10] sugerem examinar o dimensionamento da velocidade terminal com o raio das esferas para constatar que a força de arrasto é linear na velocidade para esferas metálicas em queda dentro da glicerina, com números de Reynolds muito inferiores à unidade, enquanto é quadrática na velocidade quando essas esferas caem dentro d'água, com números de Reynolds superiores a $10^{3}$. Assim, a nossa proposta se apresenta não no sentido de distinguir os termos de força com diferentes dependências da velocidade, mas de utilizálos conjuntamente. É importante salientar que, apesar da aparente simplicidade na descrição fenomenológica da força de arrasto sobre uma esfera, mesmo incorporando várias formas de dependência da velocidade, a sua fundamentação em princípios básicos da hidrodinâmica é bastante complexa e objeto de muita confusão, cuja história se estende por um longo período de discrepâncias entre os resultados teóricos, analíticos ou numéricos, e os dados experimentais [11, 12]. Além disso, evitamos a complicação que seria acrescentada caso a esfera apresentasse movimento de rotação. Quando a esfera gira em um meio viscoso, ela arrasta a camada de fluido próxima à sua superfície, alterando o campo de velocidades que teria à sua volta na ausência de rotação, e fazendo nela atuar uma força adicional, predominantemente lateral, como resultado da alteração da distribuição da pressão sobre a sua superfície, devida ao Princípio de Bernoulli, e do impulso proveniente de uma complexa modificação das velocidades na esteira do fluido posterior à esfera [13, 14]. A ação dessa força adicional sobre a translação da esfera é conhecido como "efeito Magnus" e, na prática, é bem conhecido por jogadores de tênis, na execução dos efeitos de "top-spin" ou "underspin", e dos jogadores de futebol, que fazem a bola descrever curvas em trajetórias quase imprevisíveis [15].

$\mathrm{Na}$ seção seguinte apresentamos o formalismo que fundamenta a nossa proposta. Na seção 3 apresentamos a implementação da nossa proposta no tratamento de dados de velocidade terminal por nós coletados, assim como dos dados obtidos por SCS. Na seção 4 analisamos os resultados e apresentamos as considerações finais na seção 5. No apêndice abordamos a velocidade terminal e a distância a partir da qual ela é atingida pelas esferas.

\section{Número de Reynolds, força e coeficiente de arrasto}

O comportamento do campo de velocidade $\vec{v}(\vec{r})$ e da pressão $P(\vec{r})$ em um escoamento de fluido caracterizado pela densidade $\rho$ e coeficiente de viscosidade dinâmico $\eta$, 
é governado pela equação de Navier-Stokes [1], que para um fluido incompressível é expressa por

$$
\frac{\partial \vec{v}}{\partial t}+(\vec{v} \cdot \vec{\nabla}) \vec{v}=-\frac{1}{\rho} \vec{\nabla} P+\frac{\eta}{\rho} \nabla^{2} \vec{v}
$$

Observa-se nessa equação que para a evolução da velocidade de um elemento do fluido existe a concorrência, causando efeitos opostos, entre o segundo termo do lado direito, chamado de aceleração convectiva, que faz a velocidade do elemento variar pela própria variação no campo de velocidade e que torna a equação não linear, e o último termo, em que a viscosidade age no sentido de atenuar as diferenças nas velocidades. Admitindo-se que sobre um comprimento característico $\lambda$ ocorre uma variação na velocidade da ordem de v, o termo de aceleração convectiva pode ser estimado como $v^{2} / \lambda$, enquanto a estimativa do termo viscoso fica $(\eta / \rho) v / \lambda^{2}$. A razão entre essas estimativas resulta em um número adimensional, chamado de número de Reynolds, $R=\rho v \lambda / \eta$. Logo, pequenos valores de $R$ favorecem a atenuação viscosa da diferença de velocidades, enquanto grandes valores de $R$ favorecem a aceleração convectiva, tendendo a amplificar diferenças de velocidades e até mesmo gerar turbulência. Para caracterizar o movimento de uma esfera de diâmetro $d$ dentro de um fluido, costuma-se fazer $\lambda=d$ e usar o "número de Reynolds baseado no diâmetro", $R_{\mathrm{d}}=\rho v d / \eta$. Se $\lambda$ é tomado pelo raio $r$ da esfera, tem-se o "número de Reynolds baseado no raio", $R_{r}=\rho v r / \eta$. Obviamente a relação entre esses números é $R_{\mathrm{d}}=2 R_{\mathrm{r}}$.

Quando um objeto se desloca dentro de um fluido, a força de resistência do fluido à sua passagem, chamada de força de arrasto, se deve à variação da pressão e à ação da fricção viscosa sobre a sua superfície. Observando que para números de Reynolds muito pequenos o termo de aceleração convectiva é muito menor que o termo viscoso, Stokes descartou o primeiro da equação (1) e, com essa simplificação, que elimina a sua não linearidade, calculou os campos de velocidade e pressão de escoamento de um fluido em torno de uma esfera que se move com velocidade $v$ em relação às partes distantes do fluido. Conhecido o campo de velocidade, ele pôde calcular o efeito da fricção viscosa sobre a superfície da esfera que, juntamente com a ação da variação no campo de pressão, resultaram na expressão

$$
F_{\text {Stokes }}=6 \pi \eta r v
$$

A $2^{\text {a }}$ lei de Newton aplicada a uma esfera de raio $r$ e densidade $\rho_{\text {esf }}$, em queda dentro de uma coluna de fluido de densidade $\rho$ e movendo-se com velocidade $v$ sob ação do campo gravitacional $g$, do empuxo e da força de arrasto, de módulo $F$, pode ser expressa como

$$
\rho_{e s f} \frac{4}{3} \pi r^{3} \frac{d v}{d t}=\left(\rho_{e s f}-\rho\right) \frac{4}{3} \pi r^{3} g-F .
$$

Considerando que quando a esfera atinge a velocidade terminal $v_{\mathrm{t}}$ a sua aceleração se anula e usando a força de arrasto de Stokes, dada pela expressão (2), obtemos a seguinte expressão para o coeficiente de viscosidade,

$$
\eta=\frac{2}{9} \frac{\left(\rho_{e s f}-\rho\right) r^{2} g}{v_{t}},
$$

que, em uma primeira impressão, deveria permitir de forma simples a determinação da viscosidade do fluido através da medição da velocidade terminal da esfera. No entanto, SCS examinaram o que eles chamam de "enganosa simplicidade" desse método, coletando dados de velocidade terminal por meio de filmagem de esferas de aço em queda dentro de tubos cilíndricos de diversos diâmetros, contendo glicerina, e concluíram que o método é válido apenas para números de Reynolds $R_{\mathrm{d}}<0,5$, concordando com Liao [3], ainda assim desde que se faça uma correção na velocidade devido ao efeito do diâmetro finito do tubo. A proximidade da esfera com a parede do tubo aumenta o gradiente de velocidade no fluido em torno da esfera, aumentando, consequentemente, a força viscosa e tornando necessário corrigir a sua expressão, dada pela equação (2), por um fator adimensional $\lambda_{1}$, chamado fator de Ladenburg [16], que depende da relação entre os diâmetros da esfera e do tubo, ficando

$$
F_{1}=6 \pi \eta r \lambda_{1} v
$$

Consequentemente, usando-se a velocidade terminal medida da esfera dentro do tubo, a expressão que se deve empregar para o coeficiente de viscosidade passa a ser

$$
\eta=\frac{2}{9} \frac{\left(\rho_{e s f}-\rho\right) r^{2} g}{\lambda_{1} v_{t}} .
$$

SCS argumentam que quando se obedece a relação entre o diâmetro $d$ da esfera e o diâmetro interno $D$ do tubo, $d / D<0,2$, juntamente com a condição $d<<H$, onde $H$ é a altura da coluna de líquido, um fator de correção

$$
\lambda_{1}=1+2.1 \frac{d}{D}
$$

leva a bons resultados. Essa correção pode ser associada à velocidade, significando $v_{\text {corr }}=\lambda_{1} v$ a velocidade que a esfera teria se o diâmetro do tubo fosse infinito.

Trazendo de volta o termo de aceleração convectiva, omitido por Stokes, e descrevendo a variação da velocidade do escoamento do fluido em torno da esfera como uma perturbação, em 1910 Oseen obteve uma melhor aproximação para a força de arraste, acrescentando à força de Stokes o termo

$$
F_{\text {Oseen }}=\frac{3}{8} R_{r} \times F_{\text {Stokes }}=\frac{9}{4} \pi r^{2} \rho v^{2} .
$$

Posteriormente, a solução de Oseen foi refinada por Proudman e Pearson [17], que acrescentaram às duas forças anteriores o termo

$$
F_{P P}=F_{\text {Stokes }} \times \frac{9}{40} R_{r}^{2} \ln R_{r} .
$$

A verificação da validade das expressões acima para a força de arrasto pode ser feita confrontando o coeficiente 
de arrasto, $C_{\mathrm{D}}$, com os dados experimentais. Para um objeto de seção transversal circular máxima de raio $r$, que se move com a velocidade $v$ em um fluido de densidade $\rho$, sofrendo ação da força de arrasto $F$, esse coeficiente adimensional é definido por

$$
C_{D}=\frac{F}{\frac{1}{2} \rho v^{2} \pi r^{2}}
$$

O coeficiente de arrasto exibe dependência apenas em relação à forma geométrica do objeto e ao número de Reynolds associado ao seu movimento, ficando independente da substância usada como fluido. Tomando por força de arrasto a força de Stokes acrescida das correções de Oseen e Proudman e Pearson, para uma esfera de diâmetro $d$ é previsto um coeficiente de arrasto como função do número de Reynolds baseado no diâmetro, $R_{\mathrm{d}}$, dado por

$$
C_{D}=\frac{24}{R_{d}}+\frac{9}{2}+\frac{27}{20} R_{d}^{2} \ln \left(\frac{R_{d}}{2}\right)
$$

em que o primeiro termo corresponde à força de Stokes e os termos seguintes tem correspondência com a sequência dos termos que lhe são adicionados.

Poderá ser visto adiante, que o coeficiente de arrasto obtido da correção de Oseen, usando os dois primeiros termos no lado direito da equação (11), se afasta dos dados experimentais a partir de, aproximadamente, $R_{\mathrm{d}}=3$. No entanto, a inclusão do termo de Proudman e Pearson [17], considerando os três termos na equação (11), leva a uma discordância ainda maior com os dados experimentais a partir de $R_{\mathrm{d}}=3$ [3]. A dificuldade para se obter uma expressão adequada da força de arrasto para números de Reynolds não nulos, que possa ser fundamentada pela equação de Navier-Stokes, é descrita por Veysey II e Goldenfeld [12]. A causa fundamental dessa dificuldade é que, em razão da geometria esférica envolvida, para qualquer número de Reynolds não nulo, a razão entre a aceleração convectiva e o termo viscoso da equação (1) diverge à medida que se afasta da esfera, inviabilizando o uso de métodos da teoria da perturbação e necessitando fazer uso de técnicas de renormalização. Esses autores fazem, inclusive, uma analogia do desenvolvimento do tratamento desse problema, partindo da força de Stokes, com a evolução da explicação para a crescente resolução das linhas espectrais do hidrogênio, que levou à teoria da Eletrodinâmica Quântica.

Assim, mesmo para números de Reynolds da ordem de grandeza da unidade, opta-se por trabalhar com relações empíricas que relacionam o coeficiente de arrasto ao número de Reynolds, muitas delas analisadas por Mikhailov e Silva Freire [18] na confrontação com os dados experimentais. Esses autores propõem a seguinte relação empírica para o coeficiente de arrasto,

$$
C_{D}=\frac{777\left((669806 / 875)+(114976 / 1155) R_{d}+(707 / 1380) R_{d}^{2}\right)}{646 R_{d}\left((32869 / 952)+(924 / 643) R_{d}+(1 / 385718) R_{d}^{2}\right)},
$$

que mostra-se adequada desde números de Reynolds muito baixos até $R_{\mathrm{d}} \sim 10^{5}$. O coeficiente de arrasto é praticamente constante no intervalo $10^{3}<R_{d}<10^{5}[8$, 18], indicando que a força de arrasto, nessa faixa, se reduz a uma dependência quadrática na velocidade. No entanto, a força de arrasto assim simplificada não pode ser usada na determinação do coeficiente de viscosidade pela velocidade terminal, pois o termo de Stokes, que o contém, torna-se desprezível. Uma outra forma de se perceber a dificuldade da determinação do coeficiente de viscosidade pela velocidade terminal nessa faixa é pela ausência de uma correspondência biunívoca entre o coeficiente de arrasto e o número de Reynolds, que faz com que para uma mesma velocidade terminal de uma esfera seja admitida uma extensa faixa de valores de $\eta$.

Tendo em vista que é usual empregar relações empíricas para o coeficiente de arrasto, que permite calcular a força de arrasto, propomos uma expressão para a última que incorpora, além do fator empírico de Ladenburg, $\lambda_{1}$, um segundo fator adimensional, $\lambda_{2}$, a ser obtido empiricamente e que corrige o termo adicional de força de Oseen, ficando a força de arrasto sobre a esfera dada por

$$
\begin{aligned}
F & =\lambda_{1} F_{\text {Stokes }}+\lambda_{2} F_{\text {Oseen }} \\
& =\lambda_{1} 3 \pi d \eta v+\lambda_{2} \frac{9}{16} \pi d^{2} \rho v^{2}
\end{aligned}
$$

Essa expressão permite relaxar a restrição anteriormente mencionada de se trabalhar apenas com $R_{\mathrm{d}}<0,5$, quando se usa apenas o termo de Stokes, e veremos adiante que ela é capaz, com o valor adequado para o fator $\lambda_{2}$, descrever satisfatoriamente a força de arrasto até aproximadamente $R_{\mathrm{d}}=30$. Essa última expressão para a força de arrasto implica em um coeficiente de arrasto

$$
C_{D}=\lambda_{1} \frac{24}{R_{d}}+\lambda_{2} \frac{9}{2}
$$

No caso da esfera movendo-se com velocidade terminal, a equação (3) fornece, juntamente com a equação (13), um coeficiente de viscosidade

$$
\eta=\frac{\left(\rho_{\text {esfera }}-\rho\right) d^{2} g}{18 \lambda_{1} v_{t}}-\frac{\lambda_{2}}{\lambda_{1}} \frac{3}{16} \rho v_{t} d,
$$

onde observamos que o primeiro termo do lado direito é justamente a viscosidade que seria obtida pelo método que usa apenas a força de Stokes, dada pela equação 6. Chamaremos esse termo de $\eta_{\text {Stokes }}$ e, permutando os termos na equação acima, tem-se

$$
\eta_{\text {Stokes }}=\eta+\frac{\lambda_{2}}{\lambda_{1}} \frac{3}{16} \rho v_{t} d
$$

Assim, propomos que ao tradicional procedimento de se calcular $\eta_{\text {Stokes }}$ para esferas de diferentes diâmetros por meio da velocidade terminal medida, seja acrescida a construção de um diagrama do parâmetro $\eta_{\text {Stokes }}$ em função do produto $v_{\mathrm{t}} d$. Admitindo que em uma primeira aproximação a razão $\lambda_{2} / \lambda_{1}$ seja constante, a equação (16) passa a ser representada por uma reta nesse diagrama, 
e o coeficiente de viscosidade procurado fica sendo, simplesmente, o coeficiente linear dessa reta. Se o objetivo é apenas determinar o coeficiente de viscosidade $\eta$, não é necessário se preocupar com a inclinação da reta, relacionada à razão $\lambda_{2} / \lambda_{1}$. Sendo $v_{\mathrm{t}} d=\eta R_{\mathrm{d}} / \rho$, vê-se pela equação (16) que o coeficiente de viscosidade $\eta$ converge para $\eta_{\text {Stokes }}$ quando $R_{\mathrm{d}} \rightarrow 0$, como é esperado. Enquanto o coeficiente de viscosidade $\eta$ é constante, caracterizando o fluido, o parâmetro $\eta_{\text {Stokes }}$ aparece como uma função do número de Reynolds, ou do produto $v_{\mathrm{t}} d$, em virtude da força de Stokes, quando considerada sozinha, não ser suficiente para descrever a força de arrasto para $R_{\mathrm{d}}$ $>0$. A expansão em série de Taylor do parâmetro $\eta_{\text {Stokes }}$, considerado como uma função do número de Reynolds fornece

$$
\begin{gathered}
\eta_{\text {Stokes }}=\left.\eta_{\text {Stokes }}\right|_{R_{d}=0}+\left.\frac{d \eta_{\text {Stokes }}}{d R_{d}}\right|_{R_{d}=0} R_{d}+\ldots \\
=\eta+\left.\frac{d \eta_{\text {Stokes }}}{d R_{d}}\right|_{R_{d}=0} R_{d}+\ldots
\end{gathered}
$$

e a comparação com a equação (16) justifica o fato de se poder considerar, em uma primeira aproximação, a razão $\lambda_{2} / \lambda_{1}$ como constante, independentemente da sua forma precisa. Em outras palavras, a equação (16), com a razão $\lambda_{2} / \lambda_{1}$ considerada constante, é a reta tangente à curva que representa a função $\eta_{\text {Stokes }}\left(v_{\mathrm{t}} d\right)$, quando $v_{t} d \rightarrow 0$. Obviamente poderíamos procurar por uma função que melhor representasse $\eta_{\text {Stokes }}\left(v_{\mathrm{t}} d\right)$ e, para obter $\eta$, avaliar o seu valor em $v_{\mathrm{t}} d=0$, mas o ajuste linear a ser feito aos dados experimentais é a opção mais simples.

\section{Realização experimental e obtenção do coeficiente de viscosidade}

Foram usadas cinco pequenas esferas de aço, de densidade $\rho_{\text {esfera }}=7,808 \mathrm{~g} / \mathrm{cm}^{3} \pm 0,05 \%$ e diâmetros listados na Tabela 1, para a obtenção do coeficiente de viscosidade da glicerina por meio da velocidade terminal de suas quedas dentro de tubos cilíndricos, de três diferentes diâmetros, contendo o fluido.

O primeiro tubo, de diâmetro interno $D=(31,04$ $\pm 0,03) \mathrm{mm}$, possuía cinco sensores óticos acoplados a um cronômetro digital, de forma a permitir a leitura de quatro intervalos de tempo na passagem da esfera por cada par de sensores, como mostra a Figura 1. Este equipamento destina-se especialmente para a execução desse experimento em um laboratório didático [19]. O



Figura 1: Tubo com glicerina, contendo cinco sensores óticos acoplados ao cronômetro digital, de forma a registrar quatro intervalos de tempo de passagem das esferas em queda dentro dele.

primeiro sensor foi posicionado a $13,0 \mathrm{~cm}$ de profundidade e a distância entre os pares de sensores foi de $L=(12,0$ $\pm 0,1) \mathrm{cm}$. Em acordo com os cálculos apresentados no Apêndice, a distância de 13,0 cm deve ser mais que suficiente para as esferas atingirem a velocidade terminal. Cada esfera percorreu o tubo cinco vezes, sendo que as duas esferas menores foram introduzidas usando-se um tubo direcionador, fazendo com que as mesmas caíssem de uma altura de $8,0 \mathrm{~cm}$ antes de tocar o líquido, enquanto

Tabela 1: Diâmetros internos dos tubos $(D)$ contendo glicerina, das esferas de aço usadas $(d)$, e valores das velocidades terminais $\left(v_{\mathrm{t}}\right)$ e do parâmetro $\eta_{\text {Stokes }}$ obtidos do nosso experimento.

\begin{tabular}{lcccccc}
\hline$D(\mathrm{~mm})$ & \multicolumn{2}{c}{$31,04 \pm 0,03$} & \multicolumn{2}{c}{$35,03 \pm 0,03$} & \multicolumn{2}{c}{$59,02 \pm 0,03$} \\
\hline$d(\mathrm{~mm})$ & $v_{\mathrm{t}}(\mathrm{cm} / \mathrm{s})$ & $\eta_{\text {Stokes }}(\mathrm{P})$ & $v_{\mathrm{t}}(\mathrm{cm} / \mathrm{s})$ & $\eta_{\text {Stokes }}(\mathrm{P})$ & $v_{\mathrm{t}}(\mathrm{cm} / \mathrm{s})$ & $\eta_{\text {Stokes }}(\mathrm{P})$ \\
\hline $2,998 \pm 0,005$ & $2,83 \pm 0,04$ & $9,4 \pm 0,2$ & $2,75 \pm 0,05$ & $9,9 \pm 0,2$ & $3,09 \pm 0,06$ & $9,4 \pm 0,2$ \\
\hline $5,543 \pm 0,005$ & $7,43 \pm 0,07$ & $10,7 \pm 0,1$ & $7,94 \pm 0,08$ & $10,3 \pm 0,1$ & $9,38 \pm 0,09$ & $9,7 \pm 0,1$ \\
\hline $7,997 \pm 0,005$ & $12,1 \pm 0,1$ & $12,2 \pm 0,1$ & $13,4 \pm 0,1$ & $11,5 \pm 0,2$ & $17,0 \pm 0,2$ & $10,4 \pm 0,1$ \\
\hline $9,996 \pm 0,005$ & $15,4 \pm 0,2$ & $13,8 \pm 0,2$ & $17,4 \pm 0,2$ & $12,8 \pm 0,2$ & $23,6 \pm 0,2$ & $11,1 \pm 0,1$ \\
\hline $12,687 \pm 0,005$ & $17,5 \pm 0,2$ & $17,6 \pm 0,2$ & $20,8 \pm 0,2$ & $15,6 \pm 0,2$ & $32,1 \pm 0,4$ & $12,3 \pm 0,2$ \\
\hline
\end{tabular}


as três demais esferas foram abandonadas em repouso no centro da superfície do líquido, com o auxílio de uma pinça. Devido à dificuldade de alinhamento dos sensores com a trajetória da menor esfera, para a determinação da sua velocidade terminal foram usados apenas os dois primeiros intervalos de tempo entre pares de sensores, que já indicavam uma velocidade constante. Para as demais esferas, as diferenças observadas entre os valores médios dos dois primeiros intervalos de tempo, inferiores a $5 \%$, mas superiores à incerteza que é devida ao desvio padrão da média, inferior a $1 \%$, fizeram com que fossem usados os terceiro e quarto intervalos de tempo na determinação da velocidade terminal.

O segundo tubo consistiu de uma proveta de $250 \mathrm{ml}$ de volume e diâmetro interno $D=(35,03 \pm 0,03) \mathrm{mm}$, para a qual foi improvisada a colocação de um par de sensores óticos acoplados ao cronômetro digital, separados por uma distância $L=(15,0 \pm 0,1) \mathrm{cm}$, sendo que o primeiro sensor foi posicionado a 13,0 cm de profundidade. Cada esfera foi abandonada cinco vezes no centro da superfície do líquido, com o auxílio de uma pinça.

O terceiro tubo consistiu de uma proveta de $1000 \mathrm{ml}$ de volume e diâmetro interno $D=(59,02 \pm 0,03) \mathrm{mm}$ e a cronometragem foi manual, devido à dificuldade de acoplamento dos sensores. A cronometragem iniciava-se na marca colocada a $17,0 \mathrm{~cm}$ de profundidade e terminava na marca distante de $L=(15,0 \pm 0,1) \mathrm{cm}$ abaixo da primeira. Cada esfera também foi abandonada cinco vezes no centro da superfície do líquido, com o auxílio de uma pinça.

Como a viscosidade depende fortemente da temperatura, a mesma foi monitorada, situando-se entre $23,0{ }^{\circ} \mathrm{C}$ e $24,0{ }^{\circ} \mathrm{C}$ durante a realização de todo o experimento.

As velocidades terminais obtidas para cada esfera, em cada um dos tubos, são apresentadas na Tabela 1 , juntamente com os parâmetros $\eta_{\text {Stokes }}$ calculados através da equação (6). Para esse cálculo são necessários também os valores da densidade da glicerina e da aceleração da gravidade local. Foi considerada a densidade da glicerina pura à temperatura de $25^{\circ} \mathrm{C}$, com a incerteza estimada permitindo-se uma variação de $\pm 5^{\circ} \mathrm{C}$ na temperatura $[20] \rho=1,258 \mathrm{~g} / \mathrm{cm}^{3} \pm 0,5 \%$. Quanto à aceleração da gravidade no local das medições, realizadas no campus do bairro Coração Eucarístico da PUC-Minas, em Belo Horizonte, foi considerado o valor $g=978,4 \mathrm{~cm} / \mathrm{s}^{2} \pm 0,05 \%$, inferido de medições efetuadas na região da Pampulha dessa cidade [21].
Como o interesse maior está na metodologia de tratamento dos dados, na aplicação da equação (16) para a obtenção do coeficiente de viscosidade, os dados obtidos por SCS também foram usados, estando compilados na Tabela 2. Os diâmetros das esferas e dos tubos e as velocidades medidas (não corrigidas pelo fator de Ladenburg) foram obtidos da Tabela 4 de SCS, enquanto os valores para $\eta_{\text {Stokes }}$, com a correção de Ladenburg incluída, são obtidos da Tabela 5 desses autores. Eles trabalharam no intervalo de temperatura de $23,5{ }^{\circ} \mathrm{C}$ a $24,2{ }^{\circ} \mathrm{C}$.

Com o intuito de selecionar dados adequados ao ajuste linear da equação (16), eles foram apresentados conjuntamente em um diagrama de $\eta_{\text {Stokes }}$ vs. $v_{\mathrm{t}} d$ na Figura 2. Pode-se observar que, nos dados de SCS apenas o tubo de 40,92 mm exibe o mínimo de 3 pontos aceitáveis para um ajuste linear.

Na Figura 3 são apresentados diagramas de $\eta_{\text {Stokes }}$ vs. $v_{\mathrm{t}} d$ separadamente para cada tubo, juntamente com a reta que representa o ajuste linear aos dados. Como, em acordo com o exposto ao final da seção anterior, basta procurar pela reta tangente à curva que representa a função $\eta_{\text {Stokes }}\left(v_{\mathrm{t}} d\right)$ quando $v_{\mathrm{t}} d \rightarrow 0$. Desse modo, foram excluídos do ajuste os dados de maior valor do produto

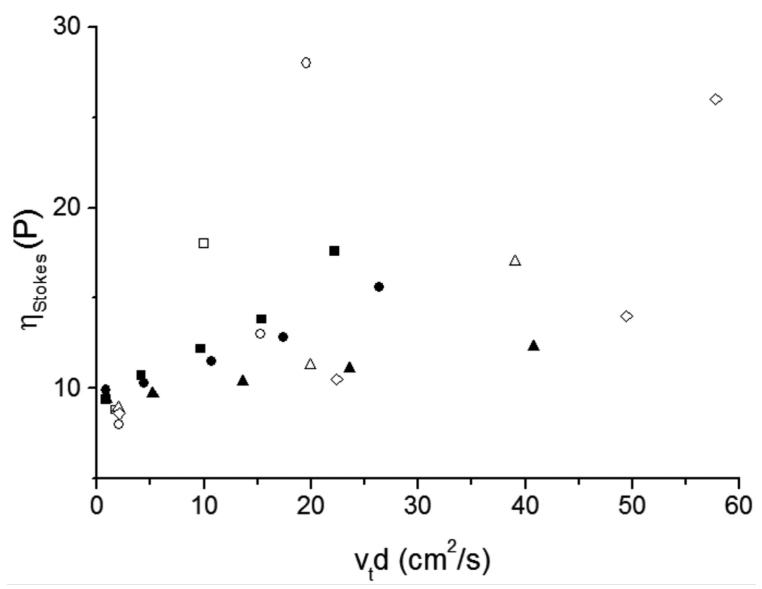

Figura 2: Apresentação conjunta dos dados das tabelas 1 e 2 em um diagrama de $\eta_{\text {Stokes }}$ vs. $v_{\mathrm{t}} d$. Os símbolos cheios representam os dados por nós obtidos, da Tabela 1, referindo-se o quadrado, o círculo e o triângulo, respectivamente, aos tubos de diâmetro interno $31,04 \mathrm{~mm}, 35,03 \mathrm{~mm}$ e 59,02 mm. Os símbolos vazios representam os dados de SCS da Tabela 2, referindo-se o quadrado, círculo, triângulo e diamante, respectivamente, aos tubos de diâmetro interno $21,60 \mathrm{~mm}, 26,29 \mathrm{~mm}, 35,5 \mathrm{~mm}$ e $40,92 \mathrm{~mm}$.

Tabela 2: Diâmetros internos dos tubos contendo glicerina, das esferas de aço usadas, e valores das velocidades terminais e do parâmetro $\eta_{\text {Stokes }}$ obtidos por SCS.

\begin{tabular}{lcccccccc}
\hline$D(\mathrm{~mm})$ & \multicolumn{2}{c}{$21,60 \pm 0,09$} & \multicolumn{2}{c}{$26,29 \pm 0,04$} & \multicolumn{2}{c}{$35,5 \pm 0,2$} & \multicolumn{2}{c}{$40,92 \pm 0,02$} \\
\hline$d(\mathrm{~mm})$ & $v_{\mathrm{t}}(\mathrm{cm} / \mathrm{s})$ & $\eta_{\text {Stokes }}(\mathrm{P})$ & $v_{\mathrm{t}}(\mathrm{cm} / \mathrm{s})$ & $\eta_{\text {Stokes }}(\mathrm{P})$ & $v_{\mathrm{t}}(\mathrm{cm} / \mathrm{s})$ & $\eta_{\text {Stokes }}(\mathrm{P})$ & $v_{\mathrm{t}}(\mathrm{cm} / \mathrm{s})$ & $\eta_{\text {Stokes }}(\mathrm{P})$ \\
\hline $3,926 \pm 0,004$ & $4,50 \pm 0,06$ & $8,8 \pm 0,3$ & $5,24 \pm 0,06$ & $8,0 \pm 0,2$ & $5,01 \pm 0,04$ & $8,9 \pm 0,2$ & $5,33 \pm 0,06$ & $8,6 \pm 0,2$ \\
\hline $9,998 \pm 0,001$ & $10,0 \pm 0,1$ & $18 \pm 1$ & $15,3 \pm 0,1$ & $13,0 \pm 0,4$ & $19,9 \pm 0,3$ & $11,3 \pm 0,4$ & $22,4 \pm 0,3$ & $10,5 \pm 0,3$ \\
\hline $15,070 \pm 0,002$ & $4,89 \pm 0,06$ & $67 \pm 3$ & $13,0 \pm 0,3$ & $28 \pm 1$ & $25,9 \pm 0,5$ & $17 \pm 1$ & $32,8 \pm 0,5$ & $14 \pm 1$ \\
\hline $20,636 \pm 0,002$ & - & - & - & - & - & - & $28,0 \pm 0,4$ & $26 \pm 1$ \\
\hline
\end{tabular}





Figura 3: Separação por tubo dos diagramas de $\eta_{\text {Stokes }}$ vs. $v_{t} d$, onde os dados obtidos do nosso experimento estão nos gráficos (a), (b) e (c), enquanto os dados de SCS estão no gráfico (d). A linha representa a reta obtida do ajuste linear aos dados, sendo que nos gráficos (a), (b) e (d) foram excluídos do ajuste os dados referentes às maiores esferas.

$v_{\mathrm{t}} d$ que destoaram significativamente de um comportamento linear, procedimento este que não enviesa os resultados de obtenção de $\eta$ pelo coeficiente linear da reta. Dentre os tubos usados por SCS, apenas foi contemplado o de diâmetro interno de 40,92 mm, pois foi o único que apresentou o mínimo de três pontos aceitáveis para um ajuste linear. Os coeficientes de viscosidade obtidos, correspondendo ao coeficiente linear das retas ajustadas, são apresentados na Tabela 3, juntamente com a inclinação das retas $(A)$, a razão $\lambda_{2} / \lambda_{1}$, obtida dessa inclinação, o número de pontos usados no ajuste e o parâmetro de correlação linear, que quantifica a qualidade do ajuste. De acordo com a equação (16), a inclinação das retas está relacionada à razão $\lambda_{2} / \lambda_{1}$ através de $A=\left(\lambda_{2} / \lambda_{1}\right) 3 \rho / 16$. É interessante observar que, para o tubo de maior diâmetro ( $D=59,02 \mathrm{~mm})$, os dados de todas as cinco esferas usadas exibem compatibilidade com uma relação linear.

\section{Análise dos resultados}

Como a viscosidade de um fluido é fortemente dependente da temperatura, é importante considerar o intervalo de temperatura em que se operou. No nosso caso, a temperatura da glicerina variou de 23,0 a $24,0{ }^{\circ} \mathrm{C}$ durante a realização do experimento, enquanto SCS reportam um intervalo de 23,5 a $24,2^{\circ} \mathrm{C}$.

Para se ter a referência de viscosidade como função da temperatura baseada na literatura, usamos a relação entre a viscosidade e a temperatura absoluta proposta por Chen e Pearlstein [22],

$$
\eta=\eta^{0} \exp \left(\frac{A}{T^{3}}+B T+\frac{C}{T}\right)
$$

para a qual Trejo González et al. [23] obtiveram, do ajuste aos dados experimentais da glicerina pura no intervalo de temperatura absoluta de 193,15 a 433,15 K, os seguintes valores para os seus parâmetros: $\ln \left(\eta^{0} / \mathrm{mPa} \bullet \mathrm{s}\right)$ $=25,8709, A=3,0942 \cdot 10^{8} \mathrm{~K}^{3}, B=-0,0327 \mathrm{~K}^{-1}$, e $C=-6291,03 \mathrm{~K}$. Essa relação, quando aplicada aos limites para os intervalos de temperatura acima mencionados, fornece os valores para o coeficiente de viscosidade apresentados na Tabela 4.

Verificamos que os valores obtidos são compatíveis com o que se espera nos intervalos de temperatura em que 
Tabela 3: Coeficiente de viscosidade $\eta$ da glicerina obtido nos diferentes tubos, de diâmetro interno $\mathrm{D}$, contendo o fluido, juntamente com a inclinação da reta tangente à curva que representaria $\eta_{\text {Stokes }}\left(v_{\mathrm{t}} d\right)$ quando $v_{\mathrm{t}} d \rightarrow 0$, a razão $\lambda_{2} / \lambda_{1}$, o número $N$ de pontos usados no ajuste linear e o parâmetro $r$ de correlação linear do ajuste.

\begin{tabular}{lccccc}
\hline$D(\mathrm{~mm})$ & $\eta(\mathrm{P})$ & $A\left(\mathrm{~g} / \mathrm{cm}^{3}\right)$ & $\lambda_{2} / \lambda_{1}$ & $N$ & $r$ \\
\hline $31,04 \pm 0,03$ & $9,3 \pm 0,2$ & $0,29 \pm 0,02$ & 1,229 & 4 & 0,99559 \\
\hline $35,03 \pm 0,03$ & $9,58 \pm 0,08$ & $0,182 \pm 0,007$ & 0,772 & 4 & 0,99838 \\
\hline $59,02 \pm 0,03$ & $9,38 \pm 0,04$ & $0,073 \pm 0,002$ & 0,721 & 5 & 0,99897 \\
\hline $40,92 \pm 0,02$ & $8,4 \pm 0,2$ & $0,10 \pm 0,01$ & 0,309 & 3 & 0,99399 \\
\hline
\end{tabular}

Tabela 4: Temperaturas referentes aos limites dos intervalos de temperatura do nosso trabalho e de SCS, e respectivos coeficientes de viscosidade da glicerina.

\begin{tabular}{lc}
\hline$T\left({ }^{\circ} \mathrm{C}\right)$ & $\eta(\mathrm{P})$ \\
\hline 23,0 & 9,51 \\
\hline 23,5 & 9,13 \\
\hline 24,0 & 8,77 \\
\hline 24,2 & 8,62 \\
\hline
\end{tabular}

se operou. O valor para $\eta$ que aparece na última linha da Tabela 3, auferido dos dados de SCS, é ligeiramente inferior aos que obtivemos e isso pode ser explicado pelo fato do intervalo de temperatura deles possuir uma temperatura média $\left(23,9{ }^{\circ} \mathrm{C}\right)$ um pouco superior à nossa $\left(23,5{ }^{\circ} \mathrm{C}\right)$.

O exame dos gráficos da Figura 3 permite inferir o limite da razão entre o diâmetro da esfera e o diâmetro interno do tubo com glicerina adequado para usar uma relação linear entre o parâmetro $\eta_{\text {Stokes }}$ e o produto $v_{\mathrm{t}} d$, associada à constância da razão $\lambda_{2} / \lambda_{1}$. O gráfico $(\mathrm{d})$, obtido dos dados de SCS, mostra que os pontos exibem linearidade até o ponto correspondente a $d / D=0,37$, mas no gráfico (b) o ponto que destoa da reta corresponde a $d / D=0,36$. No gráfico (a) os pontos exibem linearidade até $d / D=0,32$. Isso permite afirmar que a relação linear é adequada até a razão $d / D$ de cerca de $1 / 3$. Excetuando-se o tubo de maior diâmetro $(D=$ 40,92 mm), nenhum dos demais tubos de SCS possui três esferas com $d / D<1 / 3$, o que justifica a constatação anterior de não existirem para esses tubos o mínimo de 3 pontos adequados a um ajuste linear. Para o nosso tubo de maior diâmetro $(D=59,02 \mathrm{~mm})$, a maior esfera tem $d / D=0,21$, fazendo com que todos os cinco pontos apresentem pouca dispersão em relação à reta ajustada, como pode ser visto no gráfico (c) da Figura 3.

Para verificarmos a qualidade dos nossos dados experimentais, é interessante a comparação do coeficiente de arrasto deles obtido com o presente na literatura e que pode ser descrito pela relação empírica de Mikhailov e Silva Freire [18], expressa pela equação 12 .

Quando a esfera desce com velocidade terminal, a força de arrasto que nela age equilibra a combinação do peso com o empuxo, e a equação fornece

$$
C_{D}=\frac{4}{3}\left(\frac{\rho_{\text {esfera }}}{\rho}-1\right) \frac{g d}{v_{t}^{2}}
$$

A Figura 4 exibe a confrontação da curva que representa a equação (12) com os valores para o coeficiente de arrasto obtidos usando os dados de velocidade terminal medida da Tabela 1 na equação acima, nos casos em que a velocidade tanto é corrigida, multiplicando-a pelo fator de Ladenburg $\lambda_{1}$, descrito pela equação (7), como quando não sofre essa correção. O número de Reynolds foi calculado tanto com a velocidade corrigida por $\lambda_{1}$, como sem correção, e usando-se a viscosidade de 9,13 $\mathrm{P}$, correspondente ao valor médio da temperatura do intervalo em que trabalhamos, de $23,5{ }^{\circ} \mathrm{C}$. Podemos observar uma boa concordância dos pontos para os quais as velocidades foram corrigidas por $\lambda_{1}$ com a relação de Mikhailov e Silva Freire, sendo que para o tubo de maior diâmetro parece não fazer grande diferença essa correção. No entanto, veremos no Apêndice que, mesmo para esse tubo, tal correção é necessária para poder associar as velocidades medidas à velocidade que se teria no fluido longe de qualquer parede ( $D \rightarrow \infty$ ), enquanto para os tubos de menor diâmetro essa correção é insuficiente nesse sentido. Isso significa que, para os tubos de menor diâmetro, apesar dos dados referentes às velocidades corrigidas pelo fator de Ladenburg concordarem com a curva de Mikhailov e Silva Freire, eles se localizam em pontos diferentes daqueles calculados com as velocidades terminais previstas para um meio infinito que se encontram na Tabela A.1 do Apêndice, como mostra a Figura 4.

A Figura 5 apresenta os dados da razão $\lambda_{2} / \lambda_{1}$ da Tabela 3 em função do diâmetro interno do tubo, juntamente com a curva que representa um decaimento exponencial, resultante do ajuste aos dados,

$$
\frac{\lambda_{2}}{\lambda_{1}}=0,29+0,94 \exp \left[\frac{-(D-31)}{5,5 m m}\right] \text {. }
$$

Considerando que o valor assintótico, quando $D \rightarrow \infty$, do fator de Ladenburg é $\lambda_{1}=1$, a equação acima implica em um fator assintótico $\lambda_{2}=0,29$. Substituindo esses valores assintóticos nas equações $\sqrt{13}$ e $\sqrt{14}$, obtém-se a força de arrasto

$$
F=3 \pi d \eta v+0,16 \pi d^{2} \rho v^{2}
$$

e o seu respectivo coeficiente de arrasto

$$
C_{D}=\frac{24}{R_{d}}+1,31 \text {. }
$$

Espera-se, então, que essas expressões possam ser aplicadas ao movimento de uma esfera dentro de um fluido não 

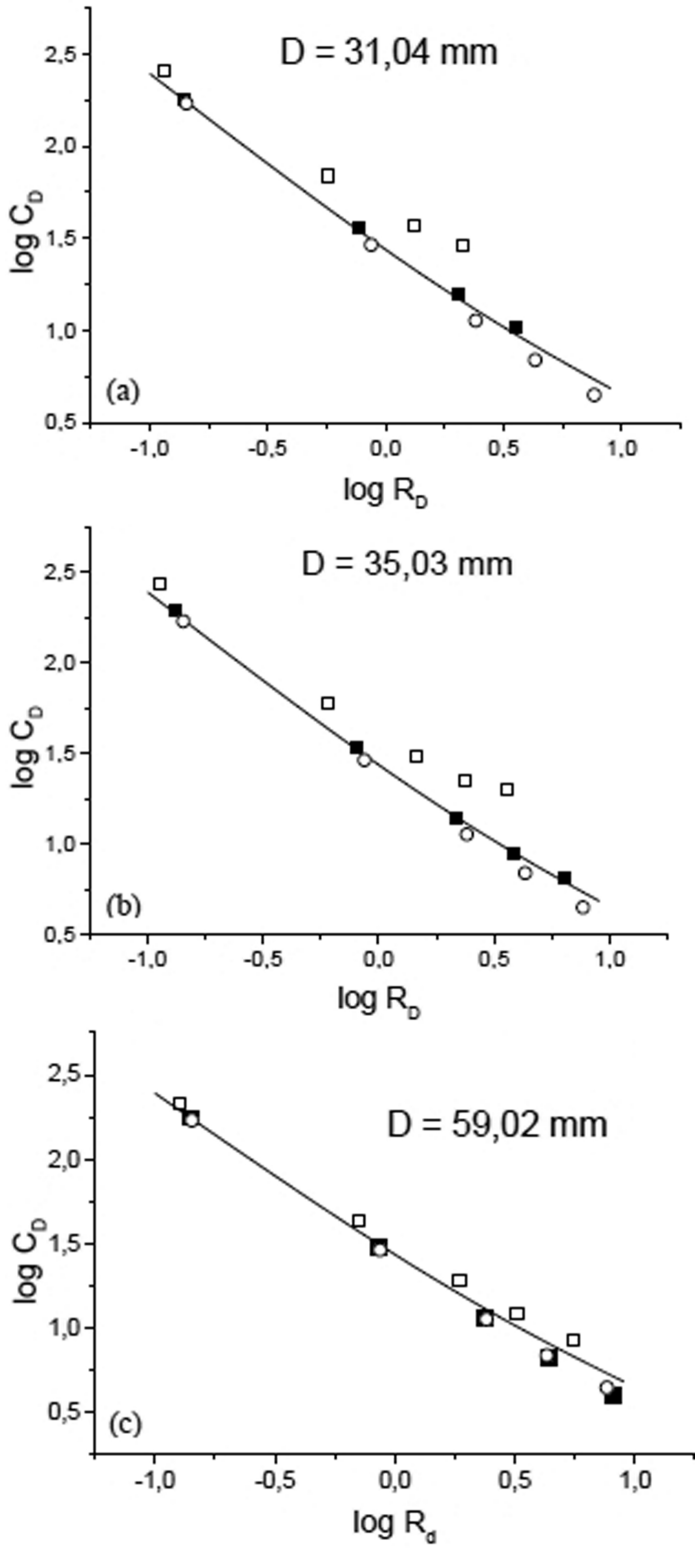

Figura 4: Gráficos em escala logarítmica (base 10) do coeficiente de arraste vs. número de Reynolds (baseado no diâmetro das esferas), obtidos das medições realizadas nos tubos de diâmetros (a) $31,04 \mathrm{~mm}$, (b) $35,03 \mathrm{~mm}$ e (c) $59,02 \mathrm{~mm}$. Os quadrados vazios referem-se aos dados calculados com a velocidade terminal igual à velocidade medida, enquanto para os quadrados cheios usou-se como velocidade terminal a velocidade medida multiplicada pelo fator de Ladenburg e para os círculos a velocidade terminal é a prevista para um meio infinito. A linha contínua refere-se à relação empírica para o coeficiente de arraste de uma esfera, obtida por Mikhailov e Silva Freire.

influenciado pelas paredes do recipiente que o contém. A Figura 6 permite a comparação do coeficiente de arrasto da última expressão com os coeficientes oriundos da força de Stokes e das correções de Oseen [5] e Proudman e Pearson [17] e com a relação empírica de Mikhailov e Silva Freire [18], que representa os dados da literatura. Podemos observar que o afastamento da relação de Mikhailov

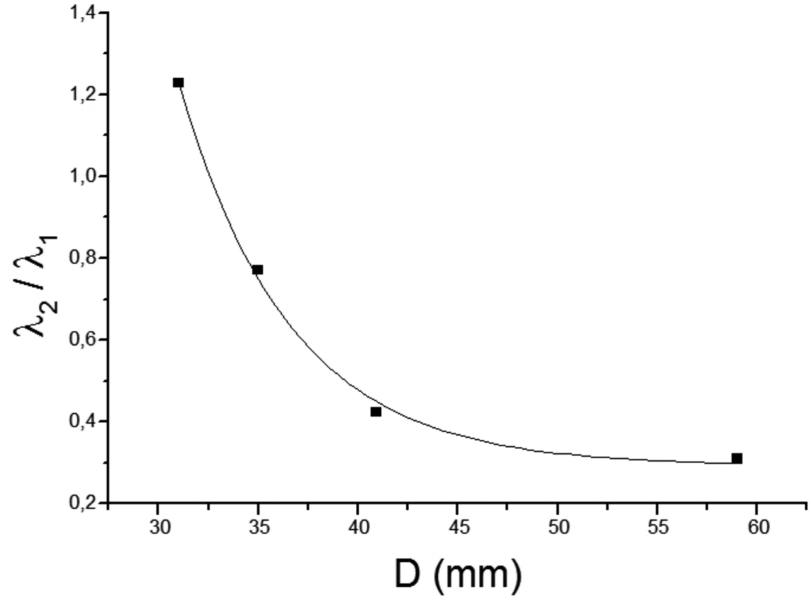

Figura 5: Gráfico da razão $\lambda_{2} / \lambda_{1}$ como função do diâmetro interno do tubo, onde os quadrados cheios referem-se aos dados da Tabela 3 e a linha refere-se à função exponencial ajustada.



Figura 6: Gráfico em escala logarítmica do coeficiente de arrasto em função do número de Reynolds, onde as linhas contínua grossa, contínua fina, tracejada, traço-ponto e pontilhada representam, respectivamente, a relação empírica de Mikhailov e Silva Freire de ajuste aos dados experimentais da literatura, a relação obtida da força de Stokes, a relação obtida de Oseen, a relação de Oseen corrigida pelo valor assintótico do fator $\lambda_{2}$ $(0,29)$, dada pela equação (22), e a relação obtida de Proudman e Pearson.

e Silva Freire ocorre aproximadamente a partir dos seguintes números de Reynolds: $R_{d} \approx 10^{0,3} \approx 2$ para a relação de Proudman e Pearson, $R_{d} \approx 10^{0,5} \approx 3$ para a relação de Oseen e $R_{d} \approx 10^{1,5} \approx 32$ para a relação que corrige o termo de Oseen pelo valor assintótico $\lambda_{2}=0,29$.

\section{Considerações finais}

No presente trabalho verificamos que a adição à força de Stokes de um termo com dependência quadrática da velocidade, como o proposto por Oseen, permite relaxar a restrição de se trabalhar com números de Reynolds $R_{\mathrm{d}}<0,5$ na determinação da viscosidade de um fluido pela medição da velocidade terminal de esferas em queda 
dentro dele, que deve ser observada quando se leva em conta apenas a primeira.

Considerando que a esfera cai dentro de um tubo de diâmetro finito, que afeta o seu movimento, assim como a força de Stokes deve ser corrigida pelo fator de Ladenburg $\lambda_{1}$, propusemos a correção do termo de Oseen por um fator $\lambda_{2}$. Dessa forma, os diferentes valores para o coeficiente de viscosidade que são encontrados quando se usa apenas a força de Stokes, $\eta_{\text {Stokes }}$, ao invés de se tornarem um empecilho e serem descartados, podem ser aproveitados na determinação do real coeficiente de viscosidade do fluido. Admitindo, em primeira aproximação, uma relação de proporcionalidade entre os fatores $\lambda_{1} \mathrm{e}$ $\lambda_{2}$, o coeficiente de viscosidade aparece simplesmente como o coeficiente linear na relação linear entre $\eta_{\text {Stokes }} \mathrm{e}$ o produto da velocidade terminal com o diâmetro das esferas, $v_{\mathrm{t}} d$, como mostra a equação (16), e não depende das especificidades de $\lambda_{2}$. Assim, para a obtenção do coeficiente de viscosidade basta construir um diagrama de $\eta_{\text {Stokes }}$ vs. $v_{\mathrm{t}} d$ e fazer um ajuste linear aos dados. Esse procedimento apresenta semelhança com o tratamento que Turian [24] fez no estudo de esferas em queda dentro de fluidos não-newtonianos, para os quais a viscosidade depende da tensão de cisalhamento à qual são submetidos. Esse autor acrescentou à força de Oseen um termo de força com dependência cúbica na velocidade, dado por $c 3 \pi v^{3} / d$, sendo c é um parâmetro empírico, constante para cada líquido, para obter

$$
\eta_{\text {Stokes }}-\frac{3}{16} \rho v_{t} d=\eta_{0}+c \frac{v_{t}^{2}}{d^{2}}
$$

onde $\eta_{0}$ é o coeficiente de viscosidade do fluido sob tensão de cisalhamento nula, que se deseja obter, e a velocidade terminal $v_{\mathrm{t}}$ se refere a um meio infinito. Analogamente ao que propomos para a obtenção do coeficiente de viscosidade, $\eta_{0}$ aparece como o coeficiente linear em um diagrama de $\left(\eta_{\text {Stokes }}-\frac{3}{16} \rho v_{t} d\right)$ vs. $\left(\frac{v_{t}^{2}}{d^{2}}\right)$. Completando a analogia, da mesma forma que no nosso caso o parâmetro $\lambda_{2}$ se relaciona ao coeficiente angular e não afeta o coeficiente linear $\eta$ no diagrama de $\eta_{\text {Stokes }}$ vs. $v_{\mathrm{t}} d$, aqui o coeficiente $c$ do termo de força cúbica na velocidade é o coeficiente angular no diagrama anterior e também não afeta o coeficiente linear $\eta_{0}$. A diferença fundamental nas duas abordagens é que enquanto Turian utiliza a força de Oseen em sua formulação original, reservando para o termo de força cúbica na velocidade a correção por um fator empírico $c$, nós já corrigimos o termo quadrático na velocidade de Oseen pelo fator empírico $\lambda_{2}$.

Aplicamos esse método aos dados de velocidade terminal de esferas de aço em queda dentro de tubos de três diâmetros internos diferentes, assim como aos dados referentes ao tubo de maior diâmetro usado por SCS, e obtivemos valores para o coeficiente de viscosidade compatíveis com os esperados no intervalo de temperatura trabalhado. Constatamos que é observada linearidade na relação entre $\eta_{\text {Stokes }}$ vs. $v_{\mathrm{t}} d$ quando a razão entre o diâmetro das esferas e o diâmetro interno do tubo é $d / D$ $<1 / 3$. Enquanto o valor assintótico, para $D \rightarrow \infty$ de $\lambda_{1}$ é 1 , os dados indicaram um valor assintótico de $\lambda_{2}$ igual a 0,29 . Usando-se esses valores assintóticos na expressão de força de arrasto proposta, chega-se a um coeficiente de arrasto que se mostra compatível com os dados da literatura até aproximadamente $R_{\mathrm{d}}=30$ que, portanto, sugere validar o uso dessa expressão de força até esse limite para o número de Reynolds, apesar de termos trabalhado no máximo até um pouco abaixo de $R_{\mathrm{d}}=10$.

Verificamos no Apêndice que a correção da velocidade medida nos tubos pelo fator de Ladenburg, descrito pela equação (7), para obter a velocidade que se teria em um meio infinito, mostrou-se adequada apenas para $d / D$ $<0,2$. É conveniente esclarecer que os fatores $\lambda_{1}$ e $\lambda_{2}$ são usados no nosso trabalho simplesmente com o propósito de uma descrição empírica para a força de arrasto, e não com o objetivo de relacionar as velocidades das esferas medidas dentro dos tubos com as velocidades que se teria em um meio infinito. O último objetivo é tema de Fidleris e Whitmore [25], que concluem por restringir ainda mais a adequação do fator de Ladenburg, na forma da equação (7), para $d / D<0,1$. Se desejássemos usar os fatores $\lambda_{1}$ e $\lambda_{2}$ para relacionar as velocidades, em seus respectivos termos de força, às velocidades $v_{\infty}$ em um meio infinito, deveríamos ter $\frac{v_{\infty}^{2}}{v^{2}}=\frac{\lambda_{1}^{2}}{\lambda_{1 \infty}^{2}}=\frac{\lambda_{2}}{\lambda_{2 \infty}}$, onde $\lambda_{1 \infty}=1$ e $\lambda_{2 \infty}=$ 0,29 são os valores assintóticos, para $D \rightarrow \infty$, de $\lambda_{1}$ e $\lambda_{2}$. Isso implicaria em $\frac{\lambda_{2}}{\lambda_{1}} \propto \lambda_{1}$, o que obviamente não ocorre, como se pode ver pela comparação das equações (20) e (7). Assim, $\lambda_{2}$ deve ser interpretado como um fator que não apenas incorpora o efeito da parede do recipiente sobre o termo de força quadrático na velocidade, mas também modifica a força de Oseen de modo a compatibilizá-la com os dados experimentais até $R_{\mathrm{d}} \approx 30$, enquanto para a expressão original dessa força essa compatibilidade fica limitada somente até $R_{\mathrm{d}} \approx 3$, como pode ser visto na Figura 6. Para o principal objetivo do presente trabalho, que é a determinação do coeficiente de viscosidade, $\lambda_{2}$ pode ser interpretado simplesmente como um parâmetro auxiliar no procedimento de extrapolação do parâmetro $\eta_{\text {Stokes }}$ para $R_{\mathrm{d}}=0$, quando $\eta$ coincide com $\eta_{\text {Stokes }}$.

Apesar da longa fundamentação desse trabalho e da extensa análise dos nossos dados experimentais, a recomendação que passamos ao laboratório didático é muito simples, consistindo apenas de um procedimento adicional no tratamento dos dados de $\eta_{\text {Stokes }}$ que usualmente já são obtidos.

Os bons resultados obtidos com o nosso tubo de maior diâmetro, mesmo fazendo uso de cronometragem manual na determinação da velocidade terminal das esferas, sugerem que a sofisticação na sua medição é desnecessária a um laboratório didático, e o que de fato deve ser priorizado é o emprego de tubo com diâmetro interno maior possível, tal que para a maior esfera usada a razão $d / D$ seja inferior a 0,2 . 


\section{Supplementary material}

The following online material is available for this article: Apêndice. A velocidade terminal e onde ela é atingida

\section{Referências}

[1] L.D. Landau and E.M. Lifshitz, Fluid Mechanics (Elsevier, Oxford, 2013), v. 6.

[2] C. Sandoval, J. Caram e J. Salinas, Revista Brasileira de Ensino de Física 31, 4310 (2009).

[3] S. Liao, International Journal of Non-Linear Mechanics 37, 1 (2002).

[4] A.A. Soares, L. Caramelo and M.A.P.M. Andrade, Eur. J. Phys. 33, 1053 (2012).

[5] C.W. Oseen, Über die Stoke'sche Formel und über eine verwandte Aufgabe in der Hydrodynamik, (Almqvist \& Wiksell, Uppsala, 1911), v. 6.

[6] D. Halliday, R. Resnick and J. Walker, Fundamentals of Physics (Wiley, New York, 2000), 6th ed.

[7] A.P. French and M.G. Ebison, Introduction to Classical Mechanics, (Kluver Academic Publisher, Dordrecht, 1986), seção 3.4 e Seção 3.5.

[8] R.D. Gregory, Classical Mechanics, An Undergraduate Text (Cambridge University Press, Cambridge, 2006), Seção 4.3 .

[9] K. Takahashi and D. Thompson, Am. J. Phys. 67, 709 (1999).

[10] J.P. Owen and W.S. Ryu, Eur. J. Phys. 26, 1085 (2005).

[11] M. Buchanan, Nature Physics 3, 213 (2007).

[12] J. Veysey II and N. Goldenfeld, Rev. Mod. Phys. 79, 883 (2007).

[13] L.J. Brigs, Am. J. Phys. 27, 589 (1959).

[14] R.G. Watts and R. Ferrer, Am. J. Phys. 55, 40 (1987).

[15] M. Duarte e E. Okuno, Física do Futebol: Mecânica (Oficina de Textos, São Paulo, 2012), p. 139-141.

[16] R. Ladenburg, Ann. d. Phys. 23, 447 (1907).

[17] I. Proudman and J.R.A. Pearson, Journal of Fluid Mechanics 2, 237 (1957).

[18] M. Mikhailov and A. Silva Freire, Powder Technology 237, 432 (2013).

[19] CIDEPE http://www.cidepe.com.br/index.php/ br/produtos-interna/viscosimetro-de-stokesmulticronometro-5-sensores-2-tubos-2123, acessado em $1 / 2 / 2017$.

[20] Glycerine Producers' Association, Physical Properties of Glycerine and its Solutions (Glycerine Producers' Association, NewYork, 1963).

[21] Domingos Sávio de Lima Soares, A Aceleração da Gravidade na Superficie da Terra, disponível em http: //www.observatorio.ufmg.br/pas46.htm, acessado em $1 / 2 / 2017$.

[22] Y. Chen and A.J. Pearlstein, Ind. Eng. Chem. Res. 26, 1670 (1987).

[23] J.A. Trejo Gonzailez, M. P. Longinotti and H.R. Corti, Journal of Chemical \& Engineering Data 56, 1397 (2011).

[24] R.M. Turian, AIChE Journal 13, 999 (1967).

[25] V. Fidleria and R.L. Whitmore, Br. J. Appl. Phys. 12, 490 (1961). 